livraisons

d'Histoire

de l'Architecture

\section{Livraisons de l'histoire de l'architecture}

$29 \mid 2015$

Ornements en architecture

\title{
Monduit s'expose : la participation de la maison Monduit aux expositions universelles.
}

The Maison Monduit on display at the International Exhibitions

Monduit stellt aus: die Teilnahme der Maison Monduit an den Weltausstellungen

\section{Caroline Mathieu}

\section{(2) OpenEdition}

Journals

Édition électronique

URL : http://journals.openedition.org/lha/470

DOI : $10.4000 /$ lha. 470

ISSN : 1960-5994

Éditeur

Association Livraisons d'histoire de l'architecture - LHA

Édition imprimée

Date de publication : 10 juin 2015

Pagination : 73-88

ISSN : 1627-4970

Référence électronique

Caroline Mathieu, «Monduit s'expose : la participation de la maison Monduit aux expositions universelles. », Livraisons de l'histoire de l'architecture [En ligne], 29 | 2015, mis en ligne le 10 juin 2017, consulté le 30 avril 2019. URL : http://journals.openedition.org/lha/470 ; DOI : 10.4000//ha.470

Ce document a été généré automatiquement le 30 avril 2019.

Tous droits réservés à l'Association LHA 


\title{
Monduit s'expose : la participation de la maison Monduit aux expositions universelles.
}

\author{
The Maison Monduit on display at the International Exhibitions \\ Monduit stellt aus: die Teilnahme der Maison Monduit an den Weltausstellungen
}

\author{
Caroline Mathieu
}

1 Glorification de l'industrie triomphante, manifestations de prestige et d'orgueil, sources de toutes les expériences, de toutes les fantaisies et véritables invitations au voyage, les expositions universelles sont nées du désir de rassembler produits et procédés de fabrications de toutes les puissances modernes, et de stimuler la concurrence. L'industrie s'exposait déjà, en des manifestations nationales, depuis la fin du XVIII siècle. La première manifestation française date de 1798 et affirme déjà « vouloir porter le coup le plus funeste à l'industrie anglaise ». Elle s'installe sur le Champ de Mars, qui va devenir l'un des lieux privilégiés des expositions universelles. De 1801 à 1849, ces présentations se renouvellent dans la cour du Louvre, sur la place de la Concorde ou les Champs-Élysées, et durent une semaine pour la première, six mois pour la dernière, ce qui sera la longueur habituelle des expositions universelles. Avec le développement des chemins de fer, du commerce et des échanges internationaux, naît bientôt l'idée d'organiser une confrontation pacifique, internationale, rassemblant commerce, industrie et beaux-arts.

C'est la Grande Bretagne, forte de sa précoce révolution industrielle et de sa stabilité politique, qui, en 1851, réunit "tous les pays d'Orient et d'Occident» en un palais exceptionnel, le Crystal Palace. Puis Paris organise sa première Exposition universelle en 1855, y adjoignant les Beaux-Arts absents à Londres. Les expositions universelles se succèdent désormais en France avec un intervalle de onze années: 1867, 1878, 1889, 1900. Vienne, bientôt les États-Unis, plus tardivement l'Italie et même l'Australie viennent enrichir et diversifier ces rencontres périodiques.

3 Les expositions universelles étaient, pour les arts appliqués à l'industrie relativement peu connus, le moyen d'exister de façon spectaculaire, comme le prouvent les photographies 
des stands de l'atelier Monduit (ill. 1) ; la création, en 1876, de L'Union centrale des arts décoratifs par Victor Champier, (1851-1929), sur le modèle du South Kensington Museum de Londres (Victoria \& Albert Museum) et celle d'un réseau d'écoles en province pour encadrer l'essor des arts appliqués à l'industrie soutenu par l'État, fait découvrir l'importance de ces métiers, de même que l'esprit des champions de l'Art Nouveau, prônant l'abolition des barrières entre arts majeurs et arts mineurs. Cependant, Champier peut encore écrire, à propos de l'Exposition de 1889 :

«Jamais, jusqu'à présent, les expositions universelles n'ont donné lieu à des ouvrages illustrés sérieusement faits, consacrés à l'étude des productions de l'art appliqué à l'industrie. Tandis que les œuvres des peintres et des sculpteurs fournissent matière à de nombreuses publications plus ou moins luxueuses, celles des fabricants, orfèvres, bronziers, céramistes, ébénistes, décorateurs, etc., - qui ont bien cependant une valeur d'art aussi ! - sont la plupart du temps traitées avec une incompréhensible négligence. De nos jours, on ignore, on méconnait les efforts réels accomplis dans les ateliers ou les manufactures pour rajeunir les formes surannées des styles passés. On ne cherche à s'enquérir ni des artistes décorateurs qui obscurément, travaillent à cette rénovation, ni des fabricants qui s'épuisent dans une lutte courageuse contre la routine de l'imitation stérile à laquelle la foule réserve ses faveurs ».

III. 1 : Le stand Mesureur et Monduit à l'Exposition universelle de 1889 à Paris

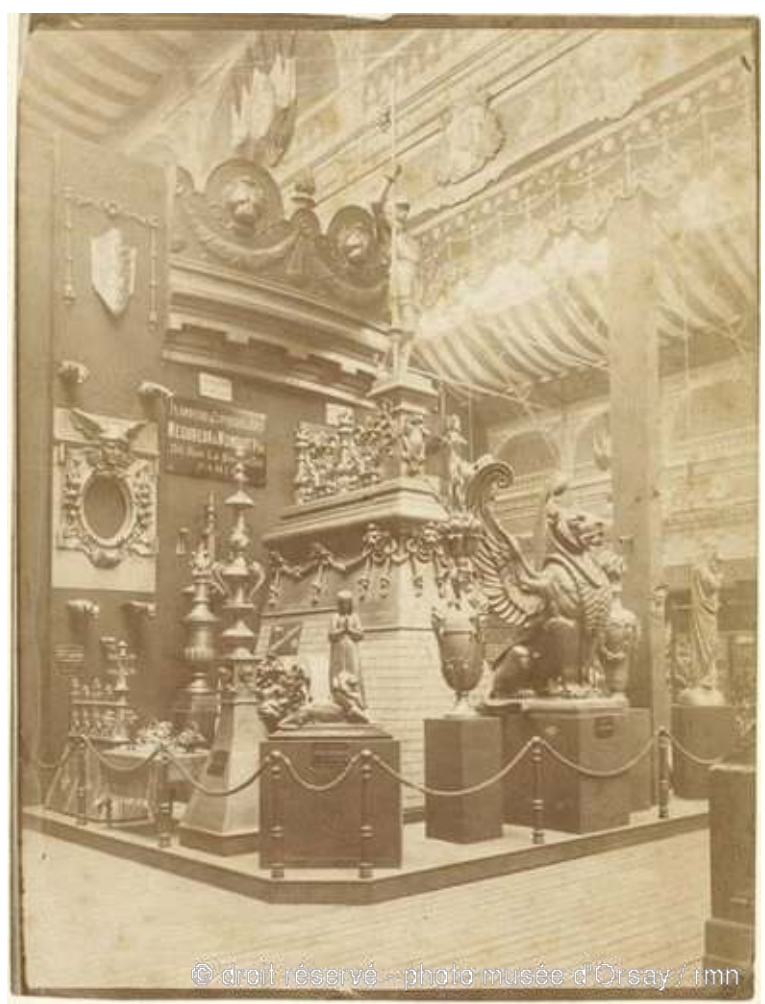

Épreuve sur papier albuminé. On reconnaît le griffon ailé de Caïn, le chevalier en armure, tous deux ornements de l'hôtel de ville de Paris, ainsi que le lion de Belfort. PHO 1996627.

(c) RMN, Musée d'Orsay, fonds Monduit, don de Mme Pasquier Monduit

Ce que Victor Champier revendique, c'est qu'à côté des architectes et des sculpteurs soit enfin reconnu le travail de l'artisan d'art, et plus précisément « l'effort du fabricant pour donner à l'art la part qui lui revient ${ }^{1}{ }^{\prime}$. 
5 C'est effectivement ce débat qui parcourt le second Empire comme la troisième République, ces difficiles rapports entre l'Art et l'industrie, dont les expositions se font régulièrement l'écho. Ainsi, en 1889, Gustave Eiffel (1832-1923) est stigmatisé comme un mercantile un « constructeur de machines » et la Tour qualifiée d' « odieuse colonne de tôle boulonnée ». Un autre exemple est particulièrement significatif: l'architecte Ferdinand Dutert (1845-1906) est présenté comme le seul inventeur de la ferme articulée du palais des machines, tandis que l'ingénieur Victor Contamin (1840-1893) qui a rendu possible cette création, est, lui, rarement mentionné. On remarque d'ailleurs, dans les diverses notes que produit pour les jurys la maison Monduit, qu'elle aussi ne laisse de revendiquer sa part légitime d'art et de création alors que justement la plomberie et la cuivrerie d'art connaissent un essor sans précédent : « Tous les monuments français, soit restaurés, soit nouvellement construits, sont dominés par des épis, des frises, ornés par des cuivres sortant de cette maison... $»^{2}$.

\section{L'excellence dès les débuts de la maison Monduit}

Bien que présents et récompensés dès $1851^{3}$, la première grande participation de la maison Monduit se fait à Londres en 1862. Associée à Béchet en 1861, la maison présente, entre autres, une réplique des statues des apôtres Matthieu et Barthélemy, parmi les douze figures qu'il avait réalisés pour la flèche de Notre-Dame de Paris (ill. 2), fabriquées sous la direction de Viollet-le-Duc, avec lequel s'étaient instaurés de vrais liens d'amitié ${ }^{4}$ et du sculpteur Geoffroy-Dechaume. En 1867, à Paris, Monduit participe à la création du palais du Champ de Mars, dessiné par l'ingénieur Jean-Baptiste Krantz (1817-1899), assisté de l'architecte Léopold Hardy et de l'ingénieur Eiffel. Il s'agit d'un édifice de plan ovale formé, autour d'un jardin, de sept galeries concentriques, dont chacune correspond à un domaine précis, tandis que chaque section rayonnante permet de parcourir l'ensemble des produits d'une nation. 
III. 2 : Charles Marville (1813-1879), Le stand Monduit et Béchet à l'Exposition universelle de Londres, 1862

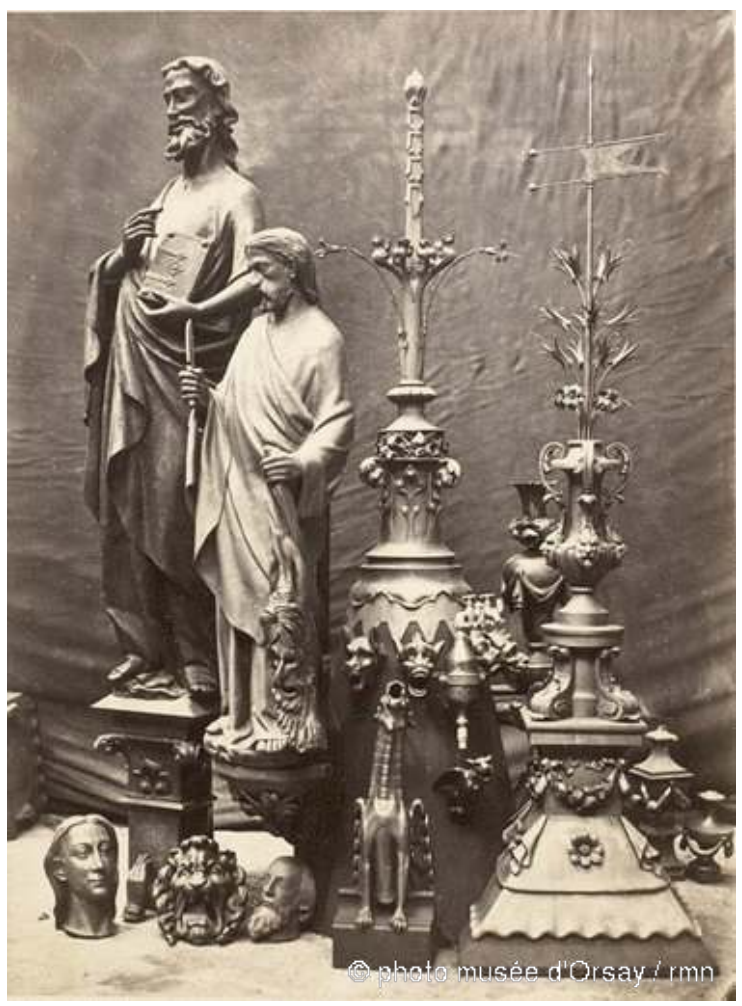

Avec les statues de Saint Matthieu pour la flèche de Notre-Dame et Saint-Barthélemy pour celle de la Sainte-Chapelle, épreuve sur papier albuminé. PHO 1996673.

(c) RMN, musée d'Orsay, fonds Monduit, don de Mme Pasquier Monduit

L'entreprise Monduit obtient pour cette participation une médaille d'or dans la classe 22, Bronzes d'art, Fontes d'art diverses, objets en métaux martelés et repoussés et fait l'objet d'une longue citation dans le rapport du jury international où sont aussi rappelés les travaux antérieurs réalisés par la maison Monduit :

"La classe 22 ne compte qu'une seule maison qui présente une série complète et très remarquable (ornements et statues) exécutés au moyen du repoussé : c'est l'établissement de MM. Monduit et Béchet. Indépendamment des produits exposés, cette maison a exécuté, pour nos monuments, d'importants travaux, parmi lesquels nous devons citer ceux du Louvre, de la Sainte Chapelle, de Notre-Dame, du dôme des Invalides, du Palais de Justice, des châteaux de Saint-Germain et de Pierrefonds etc. La plupart de ces travaux sont en plomb. Pour ceux en cuivre, nous mentionnerons seulement la statue colossale de Vercingétorix, érigée à AliseSainte-Reine (Côte d'Or) (ill. 3). Le modèle de cette statue, qui mesure 7 mètres de haut, a été composé par le statuaire Aimé Millet. L'exécution en cuivre a été commencée d'après un modèle en plâtre et a été continuée d'après dessins, en raison de modifications successives. Les accessoires ont été aussi réalisés d'après dessins. L'espace nous oblige à borner là nos citations mais, nous tenons à le dire, la maison Monduit et Béchet demeure, de tout point, digne d'encouragement $»^{5}$. 


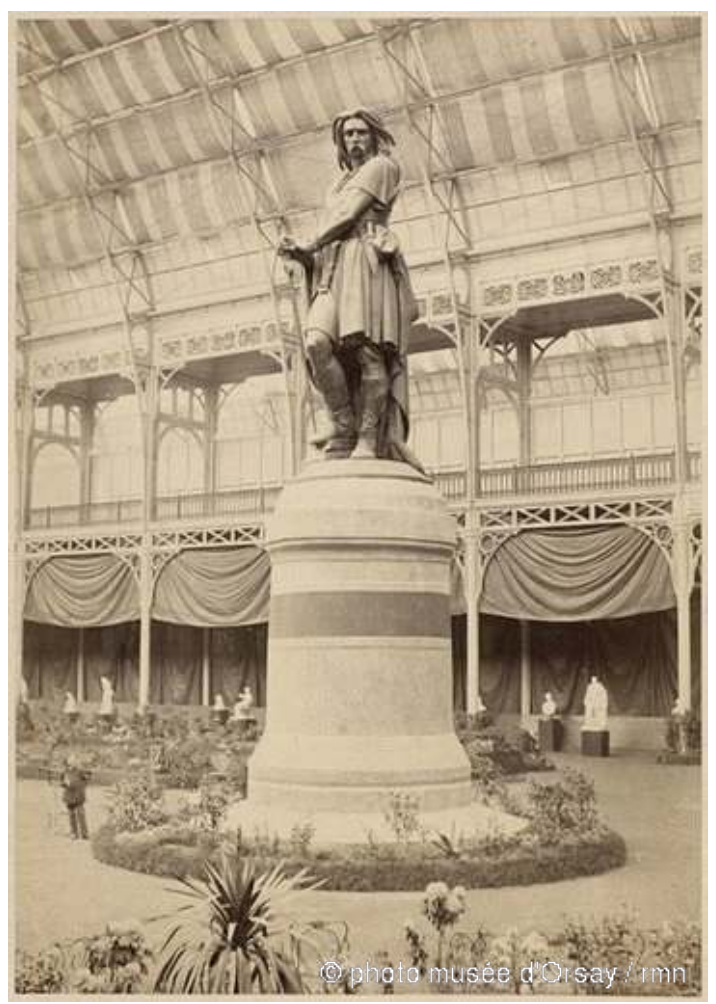

En cuivre martelé, présenté sous la verrière du palais de l'industrie, 1865. PHO 1996653.

(c) RMN, musée d'Orsay, fonds Monduit, don de Mme Pasquier Monduit

Deux autres médailles d'or s'ajoutent au prestige croissant de la maison Monduit, ainsi que des médailles remportées par les ouvriers. La prise en compte de l'équipe d'artisans, au-delà de son responsable, est particulièrement éloquente et témoigne de la reconnaissance venant s'élargir cette-fois à l'ensemble de l'entreprise. Il faut citer C-J. Morel, premier ouvrier marteleur et médaille d'or, le fidèle Albert Boignes, chef d'atelier du martelage du cuivre qui reçoit une médaille d'argent; en tout, ce sont vingt-six des collaborateurs des ateliers Monduit qui sont ainsi récompensés !6 $\mathrm{Si}$ les succès s'enchaînent à chaque participation, il convient néanmoins, à toujours veiller à convaincre le jury, et à systématiquement valoriser les progrès techniques développés par la firme et enfin de récapituler les principaux et prestigieux travaux accomplis depuis la dernière exposition. C'est aussi dans les diverses notes de l'atelier Monduit que l'on trouve de précieuses indications relatives au nombre d'ouvriers et au chiffre d'affaires. Ainsi, pour l'Exposition de Vienne en 1873, est joint un historique de la maison Monduit : «Le nombre des ouvriers employés est en moyenne de six cent cinquante pour un chiffre d'affaire de deux millions par an ", on parle aussi de la nouvelle société en commandite et de ses nouveaux associés : "Monduit, Béchet et Compagnie, avec l'adjonction de MM. Gaget et Gauthier en qualité de co-gérants ». La maison s'emploie à expliquer de façon didactique et technique les progrès accomplis dans les travaux en plomb et en cuivre :

«Dans l'exécution des travaux en plomb, Monduit et Béchet ont apporté une grande amélioration consistant dans l'étamage intérieur des pièces de plomb. Le résultat de ce mode de travail est de consolider le plomb pour pouvoir, au moyen de ferrures, fixer les pièces et éviter l'inconvénient grave du contact du chêne avec le plomb. Application en a été faite pour toutes les lucarnes du Palais des Tuileries et 
toute l'ornementation du Dôme des Invalides. Perfectionnement apporté dans le travail du cuivre depuis l'Exposition de 1867 : ainsi qu'on le verra dans l'exécution de la Vierge et des bustes composant le poinçon du chevet pour la cathédrale de Clermont (poinçon exposé à Vienne), toutes les parties du métal composant les sujets sont jointes au moyen de rivets en cuivre abreuvés dans le métal, travail ne laissant rien à désirer, puisque n'ayant plus aucune soudure, l'incendie même n'est plus à redouter, et il n'y a plus la crainte de voir les soudures se défaire et les objets se déformer $»$.

La variété et la profusion des pièces envoyées à Vienne en 1873 témoignent des qualités du travail réalisé. À ce titre les noms des architectes ou des sculpteurs figurent toujours systématiquement et viennent explicitement valoriser le travail de l'artisan. (ill. 4). La liste des œuvres est impressionnante, En plus du poinçon de Clermont-Ferrand établi à partir d'un dessin de Viollet-le-Duc, composé d'une Vierge à l'enfant en cuivre martelé, de sept bustes en cuivre dessinés par Antoine Zoegger", "le tout monté sur quatre rinceaux en plomb martelé, exécutés avec la perfection indiquée : l'étamage du plomb à l'intérieur ", on trouve la crête et le lambrequin du Palais de la Cour de Cassation à Paris ainsi que la crête et un des épis du Palais de Justice de Paris, dessin de M. Duc ${ }^{9}$ architecte. Sont exposés également « la crête avec épi exécutée au palais de M. le comte Guido Hankel à Neudeck (Silésie) ${ }^{10}$, réalisés sur un dessin de M. Lefuel ${ }^{11}$ architecte et de $V$ illeminot ${ }^{12}$, sculpteur ». L'un des "épis exécutés au château de M. le baron Seillière à Cires-les-Mello, sur un dessin de M. Destailleur ${ }^{13}$, architecte, » est aussi mentionné ; puis « un des vases exécutés au château de M. le baron de Rothschild, à Ferrières, de M. Lami $\operatorname{architecte}^{14} »$.

\section{4 : Charles Marville, Stand Monduit et Béchet à l'Exposition universelle de Vienne, 1873}



Avec les deux anges de l'église Saint-Laurent, modèle de Geffroy-Dechaume. Épreuve sur papier albuminé, et des objets provenant de l'opéra de Paris (masque, aigle), épreuve sur papier albuminé. PHO 1996622.

(C) RMN, musée d'Orsay, fonds Monduit, don de Mme Pasquier Monduit 
se poursuit avec la mention d'« un tronçon de la flèche projetée pour être exécutée à Lausanne (Suisse), sur dessin de M. Viollet-le-Duc, architecte, et de la crête de l'abbaye de Saint-Denis, de M. Villeminot, sculpteur ». Puis, suit alors l'énumération de tous les travaux importants exécutés depuis l'Exposition de 1867. Monduit reçoit une médaille d'or et Béchet la légion d'honneur. L'exposition de Philadelphie, en 1876, est trop proche de celle de Vienne, mais Monduit, Gaget-Gauthier y sont toutefois représentés par la main tenant le flambeau de la future statue de la Liberté ${ }^{15}(\mathrm{ill} .5)$.

\section{5 : Exposition de 1878, statue de l'Indépendance américaine}

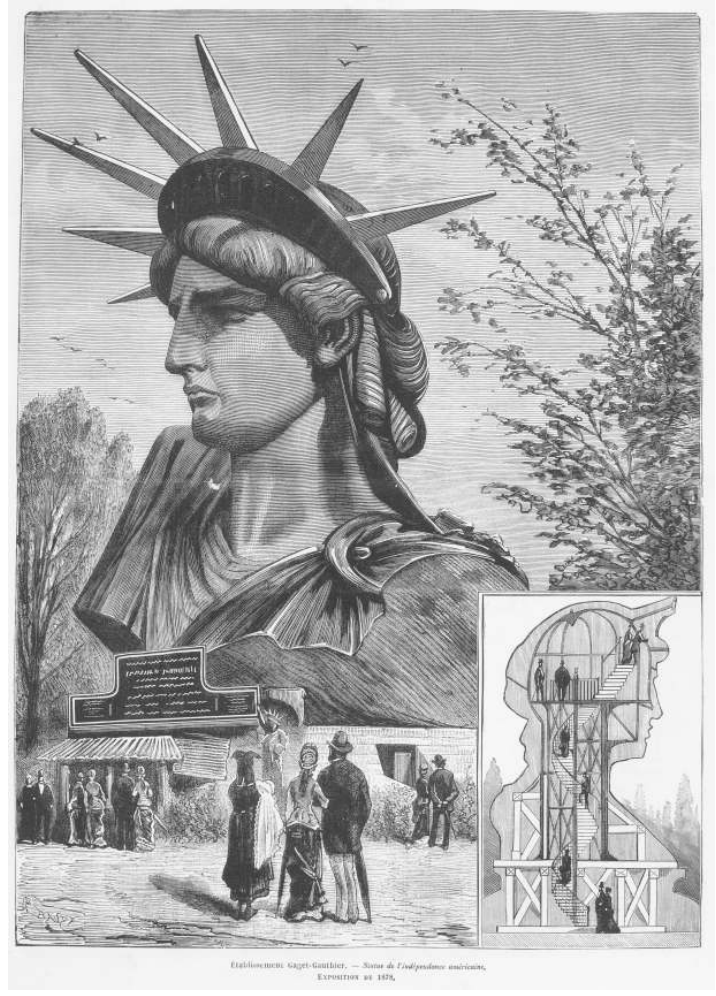

(c) Julien Turgan, Les grandes usines, bibliothèque du musée d'Orsay / Patrice Schmidt

\section{Un nouveau départ, Mesureur et Monduit}

1878 est l'exposition de la revanche :

«La France peut donner avec orgueil au monde le spectacle d'une nation si maltraitée par la conquête et les dissensions intestines, se relevant seule, en si peu de temps. Il y a à peine huit ans qu'elle a reçu un de ces coups terribles dont ses ennemis espéraient qu'elle ne se relèverait pas, et la voilà qui se montre aujourd'hui comme si elle n'avait perdu ni un soldat ni un canon et comme si elle n'avait pas eu à payer un écu de tous les milliards de sa rançon $»^{16}$.

Vaincue de la façon la plus humiliante en 1870, éprouvée par le tragique épisode de la Commune, sommée de verser à l'Allemagne cinq milliards or, la France a entrepris un effort de redressement dont l'exposition universelle concrétise la réussite. Deux grands palais sont élevés ; l'un, destiné à perdurer, sur la colline de Chaillot, le Trocadéro, œuvre de Gabriel Davioud et Jules Bourdais ${ }^{17}$, dont la maison Monduit assure l'ensemble de la couverture ainsi que la monumentale figure de la Renommée, modèle du sculpteur 
Antonin Mercié, " exécutée en cuivre repoussé au marteau dans les ateliers de MM. Monduit, Gaget et Gauthier. Cette figure dont la composition montre une fois de plus la grâce et la vigueur exceptionnelle du talent du jeune statuaire, tient d'une main plusieurs couronnes, tandis que, de l'autre, elle soutient la traditionnelle trompette. Sa dimension s'élève à $3,50 \mathrm{~m}$ et la figure est dorée ainsi que les ornements qui supportent sa base ${ }^{18}$. "

Ce sera déjà un triomphe pour l'entreprise Monduit, mais la réalisation qui remporte spectaculairement tous les suffrages est sans conteste la tête de la statue de la Liberté éclairant le monde de Bartholdi, dont la gigantesque tête, exposée au Champ de Mars, est visitée par un public très important. C'est à cette époque qu'Honoré Monduit souhaitant soutenir son fils Philippe fonde alors une nouvelle entreprise : Monduit Fils et s'associe aux frères Mesureur. Mais ce changement de statut va voir Philippe Monduit privé de la paternité de la réalisation de la statue de la Liberté. Ce changement de société explique que seuls les noms de Gaget-Gauthier soient mentionnés pour la célèbre statue, alors que l'ensemble avait été mis au point et conçu par Bartholdi et réalisé par les ateliers Monduit. D'ailleurs Turgan ne s'y trompe pas en publiant en 1881, son treizième volume des Grandes Usines dans lequel il fait l'éloge de la maison. Il consacre un chapitre entier à l'établissement Monduit et Béchet, avec les successeurs mentionnés et rappelle leurs travaux d'adduction d'eau réalisés à l'Exposition ${ }^{19}$. Une médaille d'or et un Grand prix viennent ainsi s'ajouter au palmarès de la maison et Honoré Monduit est promu au grade de chevalier de la légion d'honneur. La maison Monduit fils participe ensuite à l'exposition de Sydney en 1879 et obtient une médaille de bronze, mais déjà se profile l'Exposition d'Amsterdam en 1883. Cette fois, Monduit ne cache pas sa fierté de n'exposer que des œuvres réalisées après 1879 et encore jamais présentées et développe les nouveaux progrès atteints dans le processus de fabrication et le traitement du métal :

«Les ornements qui composent notre exposition sont tous en plomb ou en cuivre martelés à la main sans qu'aucune de leurs parties ne soit estampée à la machine, ni fondue. Les procédés que nous employons pour fabriquer ces poinçons, épis, crêtages, lucarnes et statues les rendent tout particulièrement propices à leur destination. L'épaisseur du métal employé est en moyenne de 0,0015 pour le cuivre et de 0,003 pour le plomb; une fois que les différentes pièces qui doivent composer un ornement sont battues, elles sont réunies et soudées ensemble puis montées sur une armature en fer, qui, véritable ossature, en maintient toutes les parties, les relie entre elles et permet de les fixer sur les charpentes des combles. Dans ces conditions, il est facile de se rendre compte de la très grande légèreté des ornements que nous fabriquons, ce qui est un avantage des plus sérieux puisque, en général, ils doivent être placés à une grande hauteur et que le montage et la pose en sont rendus néanmoins très facile. [...] Malgré cette très grande légèreté, nous sommes parvenus à donner à nos cuivres et à nos plombs une solidité à toute épreuve. Cela tient à ce que les opérations successives de battages et de recuit que nous faisons subir au métal, en resserre les pores et en rendent les molécules très homogènes. [...] Enfin, outre la légèreté et la solidité, un des principaux avantages de notre fabrication est un bon marché relatif ».

Suit alors la liste des pièces exposées; au Palais de Justice de Bruxelles, « un crêtage en cuivre martelé, composé de moulures, têtes de lion, guirlandes, rosaces, flammes et autres motifs. Chacun des motifs est repoussé au marteau dans une seule et même pièce de cuivre, ce malgré les saillies considérables et exceptionnelles à tout ce qui s'est fait à ce jour »; puis ce sont les lucarnes, membrons et lambrequins en plomb martelé exécutés pour l'hôtel de ville de Paris, sur des dessins des architectes Ballu et Deperthes, " véritables travaux d'art car les lignes architecturales n'ont pas exclu une ornementation composée des figures et fruits. L'examen des pièces diverses composant les motifs 
indiqués démontre que comme pour le cuivre, le martelage du métal plomb a été aussi étendu à des limites inusitées jusqu'à ce jour, l'assemblage des pièces et le mode de les fixer est d'une simplicité telle, que les points d'appui sur le chevronnage suffisent très bien pour offrir une solidité ne laissant rien à désirer : perfection du travail, légèreté des armatures, économie considérable d'ensemble d'exécution et de pose ».

Parmi les autres objets présentés, se trouve l'épi du Comptoir d'Escompte de Paris, dont l'architecte est Victor Corroyer et :

«Le modèle au $10^{\text {e }}$ d'un lion exécuté de 8 mètres de long [le lion de Belfort, de Bartholdi], [...] quelle que soit l'échelle, il est démontré que les grands artistes peuvent trouver entière satisfaction dans l'exécution en cuivre martelé de toutes leurs œuvres. [...]. De l'exécution d'une œuvre en cuivre martelé, on doit aussi reconnaître un grand avantage pour la perfection de l'œuvre: l'artiste consciencieux (tels sont tous les maîtres de ces grandes œuvres), ne sont jamais satisfaits d'eux-mêmes, et sont toujours tentés d'améliorer; avec la reproduction par le cuivre, martelé, l'artiste a cette facilité : jusque dans les dernières des limites, il peut à sa guise sur ses indications faire agir le praticien marteleur pour exécuter telles modifications qu'il peut reconnaître utiles. "

On y voit aussi l'un des sphinx du campanile de l'hôtel de ville de Paris :

« Un des quatre grands sphinx réalisés (ill. 6) justifie à tous que les engagements (de perfection de l'exécution), ont été remplis, et messieurs les architectes ont pu réaliser leur programme, réalisant une grande économie sur le système de la fonte de cuivre et aussi la réalisation d'une différence de poids considérable, point si important lorsqu'il s'agit de l'ornementation d'un campanile qui est toujours de construction très élevée et isolée ${ }^{20}$

III. 6 : Wegner et Moti, Stand Mesureur et Monduit à l'exposition universelle d'Amsterdam, 1883

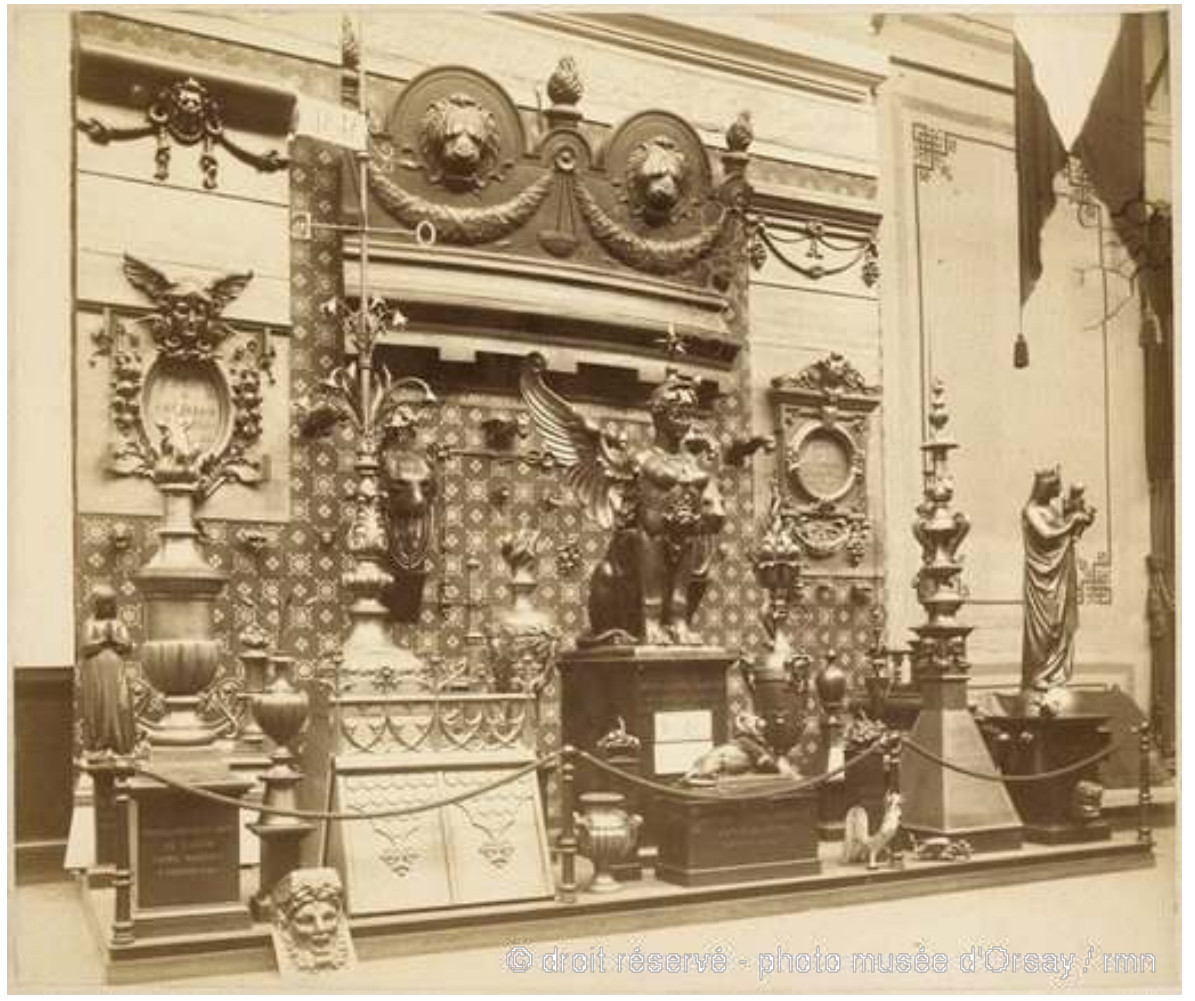

Avec la sphinge et divers ornements du nouvel Hôtel de Ville de Paris. Épreuve sur papier albuminé PHO 1996626.

(C) RMN, musée d'Orsay, fonds Monduit, don de Mme Pasquier Monduit 
Une médaille d'or et un diplôme d'honneur s'ajoutent à la consécration de la maison, signalée comme « un établissement spécial et unique en Europe $»^{21}$. À peine le temps de respirer et c'est l'exposition universelle d'Anvers de1885 ! Là encore, Monduit montre une partie des œuvres qui avait été exposée à Amsterdam, mais aussi des pièces nouvelles, comme le griffon ailé en cuivre martelé, œuvre du sculpteur Caïn, qui a travaillé avec la maison pour la reconstruction du nouvel hôtel de ville de Paris. On y admire aussi la reproduction d'une partie de son couronnement: un chevalier en armure de 2,50 m en cuivre martelé de 0,002 d'épaisseur. Les ateliers Monduit ont réalisé dix chevaliers de même dimension mais de modèles différents. On y voit également deux grandes lucarnes en plomb repoussé avec tête de Mercure et guirlandes de fruits, le tout réalisé suivant un nouveau procédé.

L'amélioration constante des processus de fabrication en cuivre et le rappel régulier des prouesses techniques constituent pour Monduit vis-à-vis de ses collègues, des organisateurs d'exposition et des spécialistes une véritable obsession, une exigence qui place en fait l'activité de l'atelier au rang d'un véritable laboratoire de recherche :

«Pour les premiers, nous avons trouvé le moyen de supprimer complètement le bois et voici comment: toutes les pièces de plomb ou de cuivre, après avoir été martelées, sont ajustées séparément au moyen d'armatures métalliques sur l'ossature en fer du bâtiment: elles sont complètement indépendantes les unes des autres et peuvent subir tous les mouvements de dilatation, non seulement sans se déchirer elles-mêmes, mais encore sans peser ou tirer sur les pièces voisines $»^{22}$.

À Anvers, la maison revient laurée de deux diplômes d'honneur, tandis que Philippe Monduit reçoit la croix de chevalier de l'ordre de Léopold. L'associé Mesureur est, lui, nommé chevalier de la Légion d'honneur.

\section{Le triomphe}

Les expositions de 1889 et de 1900 à Paris sont une véritable consécration pour Philippe Monduit. En 1889, il est chargé des toitures et de l'ornementation du Palais des BeauxArts et du Palais des Arts libéraux, «avec leurs grands dômes et leurs pavillons d'angle, leurs poinçons, arêtiers et membrons en cuivre repoussé ; les plombs fondus de la fontaine de Coutan pour la reproduction des grands dauphins (ill. 7), des vases, des cornes d'abondance etc.; et les cinquante lanternes monumentales en bronze qui servent à l'éclairage électrique du jardin central, et qui au point de vue ornemental comme au point de vue pratique, ont remporté tous les suffrages ${ }^{23}$. Cette commande de toitures vient rappeler que Monduit, de par sa formation initiale de plombier, travaille également comme entreprise de couverture. Il accumule à nouveau les récompenses dans différents domaines qui recouvrent alors aspects sociaux et hygiène - deux grands prix pour les classes 25 (bronzes) et 63 (Travaux publics et Génie civil), une médaille d'argent dans la classe 64 (hygiène) et une médaille de bronze dans l'Économie sociale et, distinction ultime, il est alors nommé officier de la légion d'honneur, mais un procès lié à un dépassement de frais généraux vient un peu assombrir sa joie.

III. 7 : L'atelier Monduit, rue Poncelet 


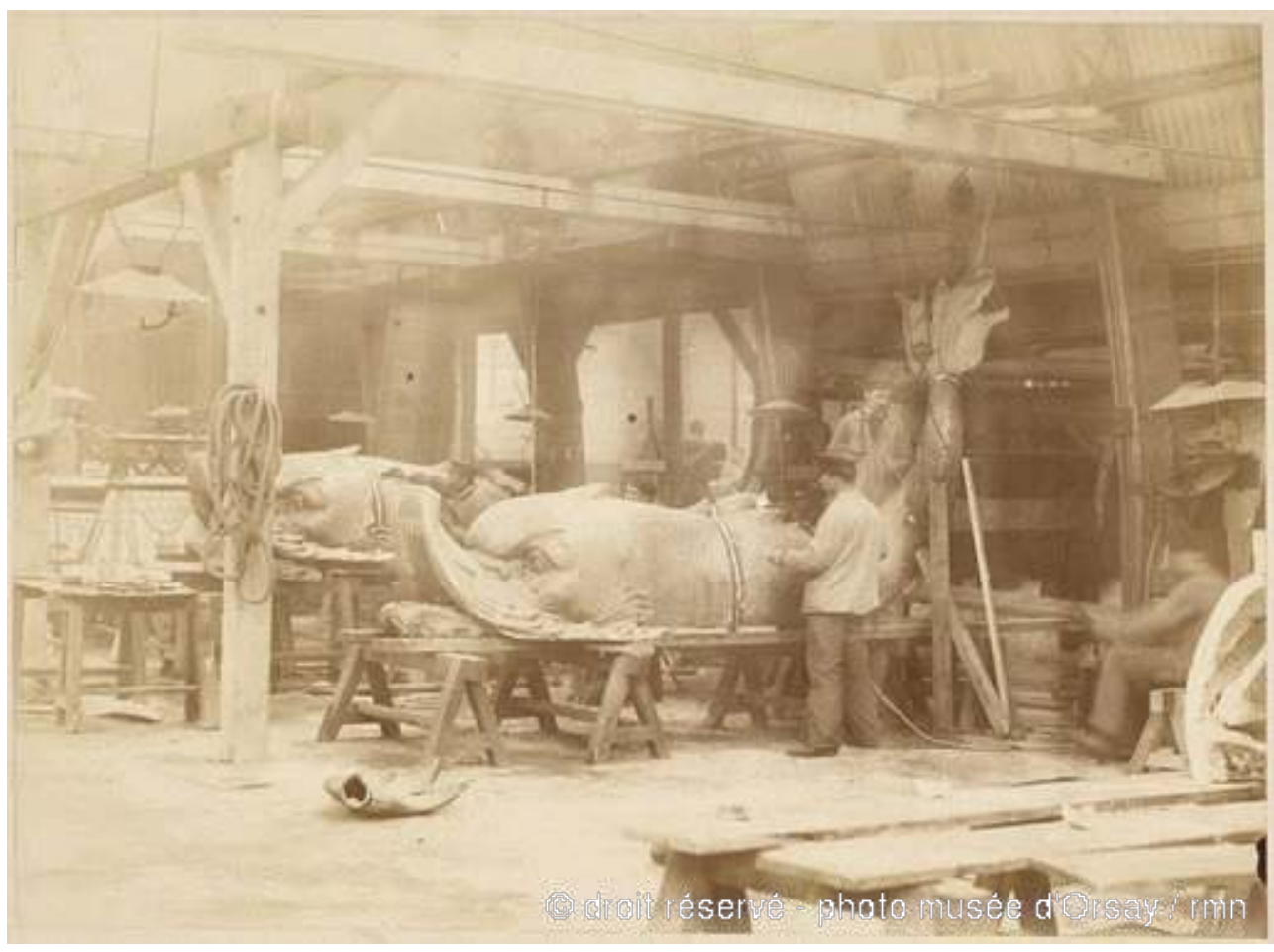

Avec les dauphins de la fontaine de Jules Félix Coutan, pour l'Exposition de 1889 à Paris, épreuve sur papier albuminé. PHO 199665.

(c) RMN, musée d'Orsay, fonds Monduit, don de Mme Pasquier Monduit

Philippe Monduit avait créé, avec trois autres entrepreneurs de travaux publics, parmi lesquels Gaget, Gauthier et $\mathrm{C}^{\mathrm{ie}}$ une sorte de syndicat:

"Sur la prière instante de M. Monduit, les associés ont bien voulu consentir à s'effacer pour ne pas nuire à son prestige [...] à le laisser seul en face de l'administration, exécuter seul les travaux, d'en établir la comptabilité, produire et encaisser les mémoires ».

Mais les associés ne sont pas du tout d'accord sur les comptes présentés par Monduit et demandent un arbitrage, confié à deux chefs d'entreprise, Leblond et Jansonie. Toute la question tourne en fait autour des frais généraux; doit-on « faire entrer en ligne de compte les frais généraux, et en fixer le quantum, si ces frais généraux doivent comprendre ceux relatifs à l'exploitation de la maison de commerce de chacun des entrepreneurs qui ont exécuté des travaux, comme le prétend M. Monduit, ce qui est contesté énergiquement par les autres syndicataires ${ }^{24}$.

Dans une lettre adressée à M. Jansonie, Monduit explique sa position :

«La question des frais généraux est tellement importante pour moi, elle me causerait un si énorme préjudice, une perte sèche si considérable, si vous la tranchiez dans un sens défavorable à mes revendications, que je ne saurais trop insister auprès de vous, pour que vous l'examiniez en homme de métier, en entrepreneur qui sait que, dans un prix de revient, la totalité de nos frais généraux doit figurer au même titre que les marchandises et la main d'œuvre. Autrement, il y a perte sur le chantier, et les autres chantiers se trouvent grevés de frais généraux encore plus considérable. Je suis sûr que, connaissant votre esprit d'équité, que vous ne consentirez pas à ce que, après avoir consacré au moins une année de ma vie à l'Exposition, après y avoir apporté tous mes soins, tous mes efforts, tout mon temps, au détriment de mes autres chantiers sur lesquels je me faisais remplacer par des commis, je me trouve dépouillé au profit de mes anciens associés qui se 
seront enrichis, tandis que moi-même, je sortirai, beaucoup moins avancé que si je ne l'avais pas entreprise, de cette malheureuse affaire ${ }^{25}$ ».

Ses adversaires lui reprochent aussi le prix du cuivre utilisé pour l'ornementation des deux palais de Formigé; Gaget et Gauthier, les anciens associés, demandent une estimation à divers estampeurs, beaucoup plus basse :

"Qu'est-ce-que ça prouve? Ce n'est un secret pour personne qu'actuellement toutes les maisons d'estampage par suite d'une concurrence insensée, traitent les affaires au-dessous de leur prix de revient. J'en ai eu cette semaine un exemple frappant. Un estampeur vient de m'enlever à Bordeaux un important travail de zinc repoussé que j'avais étudié avec un de ses confrères et il est plus que certain que l'adjudicataire ne couvrira pas la dépense. ... Leur situation est des plus mauvaises, et seules les anciennes maisons, enrichies par leurs bénéfices passés, pourront traverser cette crise $»^{26}$.

De plus, fait remarquer Monduit, c'est seulement en janvier février 1889 que l'architecte Formigé a reçu des fonds destinés à l'ornementation de ses palais, et donc, il a fallu embaucher un grand nombre d'ouvriers et leur faire faire des heures supplémentaires.

«Étant donné les délais très courts qui m'étaient accordés, je soutiens énergiquement que j'ai tiré de mes entreprises le parti le meilleur et le plus avantageux, et je n'accepte aucune réduction, ni sur la main-d'œuvre d'atelier, ni sur les frais généraux d'atelier.»

Mesureur et Monduit sont encore présents à l'exposition universelle de Milan en 1906 où un Grand prix leur est décerné. La dernière participation professionnelle de Philippe Monduit en tant que membre du jury est celle de l'exposition franco-britannique de Londres en 1908. Monduit meurt peu après en 1909:

«La mort de M. Philippe Monduit, chef de l'importante maison de ce nom (entreprise de couverture, de plomberie et de cuivrerie d'art), est une perte cruelle pour le personnel de cet établissement, pour sa corporation tout entière, où il avait acquis une place éminente et pour la Chambre de commerce de Paris, dont il était devenu le président. Notre Société le comptait parmi ses membres, et il avait doté ses employés et ses ouvriers de la participation aux bénéfices depuis 1886. Monduit joignait à ses hautes capacités beaucoup de modestie et de courtoisie, en même temps qu'une grande aménité de caractère. $»^{27}$

Heureusement, il avait reçu en 1900 une véritable consécration pour tous ses efforts, avec, entre entre autres, la réalisation des deux quadriges imaginés par le sculpteur Georges Récipon (1860-1920). Un article est prétexte à entrer de plain-pied dans l'intimité laborieuse de l'activité de l'entreprise.

«Si mon atelier vous intéresse, m'avait dit Georges Récipon, combien vous seriez plus intéressé encore par les ateliers de $\mathrm{M}$. Ph. Monduit, où mon œuvre revêt sa forme définitive, aussi légère qu'indestructible. Et tout aussitôt, un essaim de souvenirs était venu bourdonner à mes oreilles et devant mes yeux. Il s'agissait en effet de l'industrie bien française, profondément utile et très artistique de la cuivrerie d'art : l'art de représenter en lames de cuivre martelé, rivées et soudée, les œuvres les plus grandioses et souvent les plus belles de la statuaire moderne. De cet art, j'avais été le témoin voici quinze ans, alors que j'avais vu des fenêtres de mon cabinet de travail, monter et démonter pièce à pièce, la statue de La liberté éclairant le monde, de Bartholdi, ce colosse érigé aujourd'hui dans la baie de New York, et bravant de sa robe de cuivre les rafales et les embruns de l'Atlantique. Je savais que la maison Monduit- la tradition s'étant perpétuée intacte des mains du père au fils- avait à son actif, dans Paris, les œuvres les plus audacieuses, les plus géniales. Pour n'en citer qu'une et justifier mon admiration: Le génie des arts de Mercier au palais du Louvre (...) Je me hâtai donc de frapper à la porte des ouvriers 
du cuivre, modelant sous la direction de $M$. Monduit et de son très distingué chef d'atelier, M. Boignes, les figures et les chevaux des chars du Grand Palais. » $\mathrm{Au}$ milieu de la rue Poncelet, dans une halle immense comme une gare et recouverte mi-partie de tuiles, mi-partie de verres, une centaine et plus de chaudronniers travaillent et s'agitent dans la bruyante cadence, dans le strident choc des marteaux sur le souple métal. Dès les premiers pas, à droite et à gauche, voici une féerique cavalerie du plus beau ton rouge doré, de la plus haute stature (ill. 8). Ce sont sept énormes chevaux, d'aucuns manquant d'une patte, les autres de la tête, mais de corps puissant, râblé, nerveux, veines saillantes, muscles apparents ; et ces coursiers au repos font songer aux lointaines légendes, aux chevaux enchantés des conteurs des Mille et Une Nuits, des poètes arabes. Faisons quelques pas et nous serons en pleine ruche ouvrière et nous nous initierons à tous les secrets de cette industrie artistique. La voici résumée en quelques phrases par M. Monduit lui-même, que ses multiples occupations n'empêchent pas de rester le plus courtois et le plus accueillant des hommes.

Sur des modèles en fontes de fer, reproduisant exactement les modèles en plâtre livrés par le sculpteur, l'ouvrier chaudronnier commence le battage, de la feuille de métal, en se contentant d'abord de lui imprimer très sommairement les principaux reliefs au moyen de gros maillets en bois. Après un certain temps de travail, dit le silhouettage, il sent que son cuivre devient moins malléable, plus sec; afin de lui rendre sa souplesse, il le porte sur des brasiers de coke activés par un puissant ventilateur, et il le recuit. Il peut alors lui donner une nouvelle tournée de coups de marteaux qui elle-même est suivie d'une nouvelle tournée de feu, et ainsi de suite jusqu'à ce que le cuivre qui à chaque opération se rapproche davantage du modèle, sous l'action de plus en plus serrée de l'outil ait enfin réalisé la forme désirée. Chaque morceau étant battu séparément, il faut maintenant réunir entre elles les différentes pièces de la statue au moyen de brasures ou de rivures, puis monter la statue elle-même avec une mise au point très rigoureuse. Une fois la statue assemblée, on la garnit intérieurement d'une légère armature en fer qui suit très fidèlement les principaux contours de cuivre et qui constitue une véritable ossature. Cette ossature est elle-même reliée à un arbre principal en fer, qui est fixé solidement sur l'édifice. Quand nous aurons dit que les lames de cuivre employées ont généralement une épaisseur de un millimètre et demi, le lecteur se rendra compte de l'extrême légèreté de cette statuaire de cuivre, à laquelle le battage, le martelage, en resserrant toutes les molécules donnent une cohésion, une solidité incroyable ". C'est bien là une des plus admirables applications de la Science pour servir l'Art en ses fantaisies chimériques. Et nous sommes sûrs que confiés en d'aussi bonnes mains, les «quadriges » du Grand Palais braveront les intempéries des saisons, la morsure des années et longuement témoigneront de la puissante envolée, de l'audace et de la force de la sculpture française à l'aube du vingtième siècle $^{28} »$. 
III. 8 : L'atelier Monduit avec la fabrication des chevaux des quadriges de Récipon pour le Grand Palais, exposition universelle de 1900

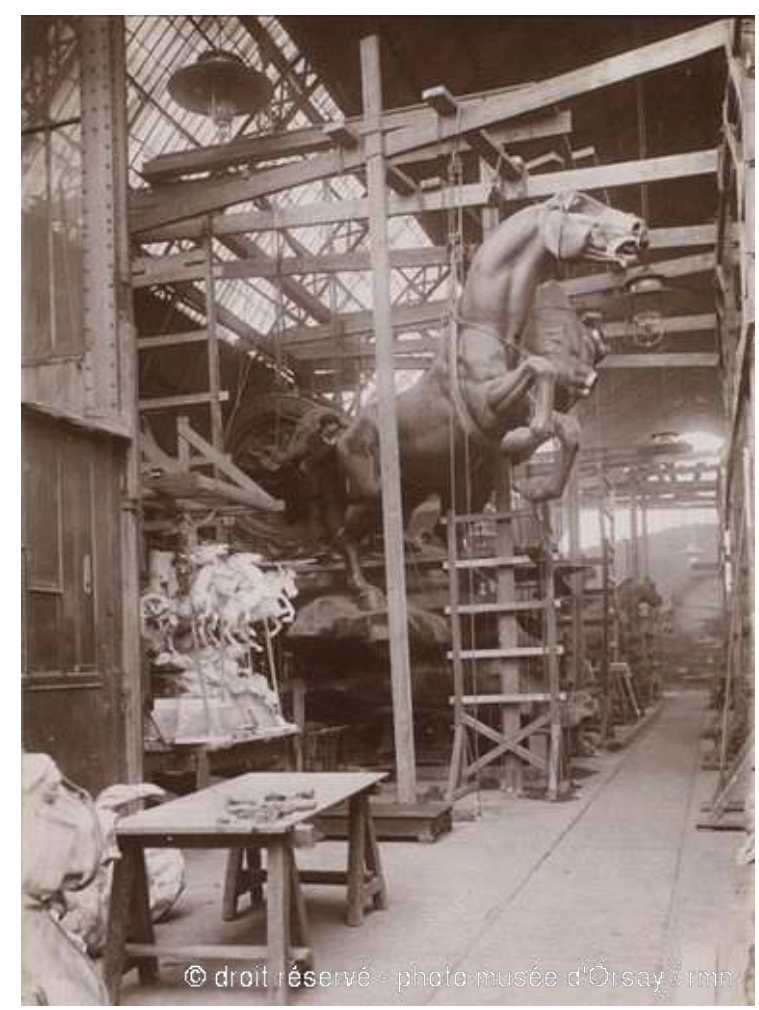

Épreuve sur papier albuminé. PHO 2001310.

(c) RMN, musée d'Orsay, fonds Monduit, don de Mme Pasquier Monduit.

C'est aussi une vivante illustration d'une aventure entre art et industrie, révélatrice de tout l'esprit du XIX siècle, architectural, politique, social et de tout ce savoir-faire, de cet enthousiasme positiviste qui caractérisent cette époque inventive et prodigieuse.

\section{NOTES}

1. Victor Champier, «Les industries d'art à l'Exposition universelle de 1889 », dans le supplément à la Revue des Arts décoratifs, novembre et décembre 1890, fascicule 15, Paris, 1890. Champier a fondé cette revue en 1880, publiée sans interruption jusqu'en 1902.

2. Julien Turgan, Les Grandes Usines, études industrielles en France et à l'étranger, Paris, Calmann-Lévy, XIII, 1881, p. 1 à 16.

3. En 1855, Durand, dont Monduit rachète le fond en 1860, est primé et fait chevalier de la légion d'honneur.

4. Louis-Honoré Monduit est parmi les rares personnes qui assistent à l'enterrement de l'architecte, à Lausanne, en 1879. 
5. Rapport du Jury International, publié sous la direction de Michel Chevalier, tome III, groupe III, classes 14 à $26, p$. 307. Le rapport était confié à des experts, et pour le groupe bronzes d'art, fontes d'art diverses, objets en métaux repoussés le fondeur Ferdinand Barbedienne avait été sollicité.

6. Exposition universelle de 1867, Catalogue officiel des exposants récompensés par le jury international, Paris, E. Dentu libraire-éditeur, 1867, p. 19 et 20.

7. Musée d'Orsay, fonds Monduit. Le musée d'Orsay a bénéficié de la générosité de Mme Gabrielle Pasquier-Monduit, à plusieurs reprises, par un don de lavis pour les catalogues Monduit, de calques, de photographies, de médailles et de nombreuses archives, réunis sous le titre : Fonds Monduit.

8. Antoine Zoegger, 1829-1885.

9. 1802-1879.

10. Le comte Henckel von Donnersmark avait épousé la célèbre Païva et fait construire l'hôtel particulier situé au 25 avenue des Champs Elysées ; le musée d'Orsay conserve un dessin de Lefuel pour le château de Neudeck (ARO 1982-3), Neudeck est le nom allemand, Swierklaniec est le nom polonais; il fut rasé par le régime communiste en 1965. Le musée possède aussi une des quatre consoles du grand salon de l'hôtel des Champs-Elysées. (OAO 1323), une autre se trouve au Musée des Arts décoratifs.

11. Hector Lefuel, 1810-1881.

12. Louis Villeminot, 1826-1914.

13. Hippolyte-Alexandre Destailleur, 1822-1893.

14. Eugène Lami, 1800-1890, peintre et décorateur.

15. Le rapport du jury international mentionne la faible participation de la France, et mentionne qu'aucun fabricant de métaux repoussés n'y est présent.

16. Le Globe, journal de Londres. Cité dans L'Exposition de Paris (1878), journal hebdomadaire, $\mathrm{n}^{\circ} 9$.

17. Remplacé par le palais de Chaillot, élevé pour l'exposition Internationale de Paris, 1937, mais dont une partie a été englobée dans le nouvel édifice

18. Exposition universelle de 1878, Le Palais du Trocadéro, Paris, Ve A. Morel et Cie, Libraireséditeurs, p. 129.

19. Julien Turgan, op.cit., p. 1 à 16.

20. Note présentée par MM. Mesureur et Monduit fils à Messieurs les membres du jury de la classe 46, Exposition d'Amsterdam, musée d'Orsay, fonds Monduit, avec corrections de Philippe Monduit.

21. "Les vainqueurs de l'exposition de Vienne, membres de l'Union Centrale», dans Bulletin de l'Union centrale, Revue mensuelle des Beaux-Arts appliqués à l'Industrie, juin 1875, 1ère année, $n^{\circ} 11$, p. 289.

22. Note présentée à Messieurs les membres du jury par Mesureur et Monduit Fils, groupe 2, classe 20, musée d'Orsay, fonds Monduit. Ces notes sont très précieuses ; à chaque fois est joint un récapitulatif des derniers travaux importants menés par l'atelier.

23. Note présentée par Ph. Monduit à l'appui de sa demande de proposition pour la croix de la légion d'honneur; c'est le jury des récompenses de la classe 25 qui demande cette distinction pour Philippe Monduit. 
24. Musée d'Orsay, fonds Monduit ; malheureusement, je n'ai pas trouvé la conclusion de ce procès, mais tout porte à croire que Monduit l'a gagné. L'association avec Mesureur se termine aussi par un procès, gagné par Monduit fils en 1913, alors que la maison est administrée par l'architecte Léon Pasquier, père de Mme Gabrielle Pasquier-Monduit.

25. Musée d'Orsay, fonds Monduit.

26. Note complémentaire présentée à Messieurs les arbitres sur la question des travaux exécutés dans mon atelier, 15 juillet 1892, musée d'Orsay, fonds Monduit.

27. Bulletin de la participation aux bénéfices publié par la société pour l'étude pratique de la participation du personnel dans les bénéfices, reconnue d'utilité publique par décret du 12 mars 1889, s. 1., 1909, Chaix Imprimeur, p.221

28. Le Monde Illustré, 23 décembre 1893, p. 449

\section{RÉSUMÉS}

Les expositions universelles au XIX ${ }^{\mathrm{e}}$ siècle et au début du XX $\mathrm{XX}^{\mathrm{e}}$ siècle ont entre autres excellences contribué à faire connaître le prestige de certains artisans. A ce titre, la maison Monduit figure incontestablement parmi l'une des plus prestigieuses et des plus représentatives de son temps. Grands plombiers d'art, ils ont participé régulièrement aux grandes expositions universelles en France et à l'étranger, mais la reconnaissance de leur talent sur la scène internationale ne fut pas si aisée. Il a fallu que la maison Monduit, relayée par la presse savante et populaire et par les connaisseurs et les experts, ne cesse, dans le cadre des expositions, de rappeler la diversité et le nombre d'œuvres fabriquées et surtout s'emploie à montrer les innovations apportées dans la technique du plomb et du cuivre. Il a fallu qu'elle rappelle que son travail ne s'exerçait pas de façon autonome, mais en collaboration et sous la maîtrise d'œuvre d'architectes parmi les plus talentueux et au service des sculpteurs les plus renommés. Ce double parrainage lui a assuré ainsi qu'à ses ouvriers une large part de sa notoriété et de son rayonnement à l'étranger. L'œuvre réalisée et fabriquée par la maison Monduit résonne enfin en écho sur le long débat engagé tout au long du XIX ${ }^{\mathrm{e}}$ siècle sur les délicates limites entre l'art et la technique.

Among many other advantages, the International Exhibitions of the XIX ${ }^{\text {th }}$ century and the $\mathrm{XX}^{\text {th }}$ century contributed to enhance the publicity of a few craftsmen. From this point of view, the Maison Monduit stands among the most prestigious and representative companies. As artistic plumbers, they regularly took part in the International Exhibitions in France and abroad, but the acknowledging of their skills on the international stage was not easy. Supported by scientific and popular papers, by experts and scientists, the Maison Monduit had to keep reminding the diversity and the number of her works of art, and moreover shows the innovations allowed by the cupper and lead technic. She had to remind her work was not independent, but in close collaboration and under the direction of the most gifted architects to promote the works of wellknown sculptors. This double patronage let her (as well as her craftsmen) get awareness and publicity. The work designed by the Maison Monduit meets finally success after the very long debate which occurred all along the XIX ${ }^{\text {th }}$ century on the limits between art and technic.

Die Weltausstellungen des ausgehenden 19. und beginnenden 20. Jahrhunderts haben entscheidend zur Bekanntheit und zum Prestige verschiedener Kunsthandwerksbetriebe 
beigetragen. Die Maison Monduit nimmt darunter eine herausragende und für ihre Zeit besonders repräsentative Stellung ein. Als ausgezeichnete Bleigießer waren sie regelmäßig auf großen Ausstellungen im In- und Ausland vertreten, auch wenn sie auf internationaler Bühne nicht dieselbe Anerkennung wie in Frankreich fanden. Über die Fachpresse als auch über zahlreiche berufliche Kontakte strebte der Betrieb Monduit stetig nach einer weiteren Verbreitung und Bekanntmachung seiner Produkte und präsentierte anschaulich seine Erfindungen auf dem Gebiet der Blei- und Kupfertechnik. Dabei war Monduit besonders daran gelegen, die Zusammenarbeit mit renommierten Architekten und Bildhauern herauszustellen, woher das Haus schließlich einen großen Teil seiner Bekanntheit und seinem Ruhm im Ausland verdankt. Das Werk der Maison Monduit findet darüber hinaus Niederschlag in den über das gesamte 19. Jahrhundert andauernden Debatten über die heiklen Grenzziehungen zwischen Kunst und Technik.

\section{AUTEUR}

\section{CAROLINE MATHIEU}

Caroline Mathieu est au musée d'Orsay dès 1980 et travaille sous la direction de Michel Laclotte, à l'élaboration du programme scientifique du musée. Responsable des collections d'architecture, elle a participé aux expositions Viollet-le-Duc (1980, sous la direction de B. Foucart), Le Japonisme (1988), Paris - Barcelone (2002); au musée, elle a été commissaire de l'exposition: 1889. La Tour Eiffel et l'Exposition universelle, 1989, et de projets soulignant les liens entre art et industrie: Les Schneider, Le Creusot, 1995, La maison de Wendel, 2004, Saint Gobain, 2006. Elle a organisé des expositions contribuant à une meilleure connaissance des collections et de l'art de la seconde moitié du XIX ${ }^{\mathrm{e}}$ siècle dans le monde, au Japon, aux États-Unis, en Corée, en Australie, en Chine, à Singapour, au Brésil. C. Mathieu a publié textes et articles (L'art du XIX ${ }^{e}$ siècle, 1990), et, sur les collections du musée : guides du musée et des collections, 1986, 2004 et 2012, La peinture au musée d'Orsay, 2003; Orsay. L'Architecture, 2003. En 2009, elle a organisé pour la ville de Paris la rétrospective consacrée à Gustave Eiffel, le magicien du fer, présentée à l'hôtel de ville. En 2010, elle a assuré le commissariat, avec Akiya Takahashi, de l'exposition Édouard Manet et le Paris Moderne, au musée Mitsubishui Ichigokan de Tokyo, et, en 2011, celui de l'exposition Paris au temps des Impressionnistes à l'Hôtel de ville de Paris. Cette même année, elle a organisé l'exposition Rêve et réalité à Séoul en Corée et à Singapour. Cette année, elle est commissaire de l'exposition Au-delà de l'Impressionnisme : naissance de l'art moderne au National Museum of Korea à Séoul, comprenant 173 œuvres de toutes techniques, puis de l'exposition Naissance de l'Impressionnisme. La liberté de peindre, au National Art Center de Tokyo. Elle a enseigné à l'École du Louvre et à l'Institut national du patrimoine. Adresse électronique : caroline.mathieu@musee-orsay.fr 